\section{Planejamento familiar: a autonomia das mulheres sob questão}

\section{Family planning: a matter of women's choice}

\author{
Ana Maria Costa 1 \\ Dirce Guilhem 2 \\ Lynn Dee Silver 3
}

Abstract

Objectives: to investigate women's access to contraceptive methods.

Methods: inquiry through a self-applied survey of a random sample (divided into geographic regions and population size). The study comprised all 5507 Brazilian municipalities.

Results: an outstanding number of municipalities place a high priority on family planning in relation to geographic region and size. As for contraception availability, $79.5 \%$ of 5358 municipalities do not provide diaphragms, and only $6.6 \%$ meet over $75 \%$ of the demand. In contrast, more than $75 \%$ of the demand for injectable contraceptives is met in $13.4 \%$ of 5314 municipalities. Seventy two and nine percent (72.9\%) of all Brazilian municipalities does not offer fertility treatment, and only $5.9 \%$ of the municipalities provide contraceptives to over $75 \%$ of the women seeking this service.

Conclusions: findings indicate inconsistency between education and routines of family planning, quantitative and qualitative access restrictions to contraceptive methods and insufficient care for infertility. This scenario limits women freedom of choice.

Key words Family planning services, Women's health, Contraception

\section{Resumo}

Objetivos: verificar as condições de acesso das mulheres às atividades de atenção e aos métodos contraceptivos no Brasil.

Métodos: inquérito exploratório por questionário auto-aplicado em amostra de municípios, probabilística, aleatória e estratificada por região geográfica e por dimensão populacional.

Resultados: os resultados, obtidos por aplicação de fatores de expansão da amostra, abrangem 5507 municípios. Destaca-se a elevada priorização do planejamento familiar associado à região geográfica e ao tamanho do município. A estimativa de cobertura atendida está associada à modalidade de gestão.O diafragma não é oferecido por 79,5\% de 5.358 municípios e apenas 6,6\% deles atendem à demanda por diafragma superior a $75 \%$, contrastando com os injetáveis, para os quais $13,4 \%$ de 5314 municípios conseguem atender a mais que $75 \%$ da demanda. A atenção à infertilidade não é realizada em $72,9 \%$ dos municípios brasileiros $(n=5349)$ e, em apenas $5,9 \%$ deles, são atendidas demandas superiores a $75 \%$.

Conclusões: os resultados encontrados denunciam a dissociação entre as práticas educativas e a rotina de atenção ao planejamento familiar; restrições qualitativas e quantitativas de acesso aos métodos contraceptivos e ainda a baixa oferta de atenção à infertilidade. Ficam evidentes as condições de comprometimento do exercício da autonomia das mulheres quanto a livre escolha dos métodos contraceptivos.

Palavras-chave Serviços de planejamento familiar, Saúde da mulher, Anticoncepção 


\section{Introdução}

No Brasil, o tema planejamento familiar sempre suscitou polêmicas. Ao longo dos anos 70 o debate em torno do controle demográfico no Brasil encontrava-se acirrado e polarizado. A fragilidade política com que o Ministério da Saúde enfrentava o tema do planejamento familiar criou um vácuo institucional do Estado e favoreceu a ação das instituições de cunho controlista, que agiam de forma desordenada em todo o território nacional.1,2,4 Dentre essas, a Sociedade Civil de Bem-Estar Familiar no Brasil (BENFAM) e o Centro de Pesquisas de Assistência Integrada à Mulher e à Criança (CPAIMC) foram as de maior relevância. ${ }^{1}$

A ambigüidade do governo brasileiro em relação ao planejamento familiar é discutida por Rocha, 3 segundo a qual a posição de cautela do governo em relação à política social de controle da fecundidade convivia com a posição permissiva acerca da atuação de entidades privadas. A autora atribui essa situação às objeções de setores do Estado e da sociedade às políticas de controle demográfico.

No início dos anos 80, a radicalização do discurso dos militares em relação ao tema do controle demográfico foi seguida da reação indignada de novos atores sociais, incluindo o movimento feminista, recém-articulado no país. Doravante, manifestando suas preocupações com as mulheres, objeto específico destas políticas de população, as feministas, mesmo pouco numerosas e concentradas em poucas cidades brasileiras, introduziram no debate posições firmes sobre as suas aspirações.

Não desejando ser identificado com os argumentos de controle populacional, o movimento feminista clamava pela autonomia das mulheres na escolha dos seus destinos em relação à procriação. 5 Além das fortes críticas lançadas às instituições que atuavam no controle demográfico, o movimento contrapunha-se também aos argumentos pró-natalistas de ocupação do território nacional e outros argumentos afins. $\mathrm{O}$ movimento feminista propunha um desfecho às manipulações dos controlistas e dos natalistas sobre o corpo feminino. ${ }^{6-7}$

Importante ressaltar que, desde os anos sessenta, as mulheres brasileiras vinham processando a ruptura com o clássico e exclusivo papel social que lhes era atribuído pela maternidade, introduzindo-se no mercado de trabalho e ampliando suas aspirações de cidadania. Controlar a fecundidade e praticar a anticoncepção passaram a ser aspirações das mulheres, assim como a vivência plena da sexualidade, desvinculando a maternidade do desejo e da vida sexual.5 Essa conjuntura implicou a necessidade de políticas que permitissem o acesso aos métodos contraceptivos.

Em 1983, nesta complexa conjuntura em que o conjunto de argumentos científico-políticos fundamenta os distintos interesses em jogo na questão populacional, o governo brasileiro mobilizou-se por meio do enfrentamento da questão do planejamento familiar e suas repercussões para o âmbito da Saúde. O Ministério da Saúde foi convocado a apresentar uma proposta de política concreta sobre o tema, e formulou o Programa de Assistência Integral à Saúde da Mulher (PAISM), enquanto o Congresso Nacional realizava uma Comissão Parlamentar de Inquérito para avaliar o crescimento demográfico no país. Esse movimento do Governo e do Poder Legislativo tinha ainda o objetivo de construir a posição brasileira a ser apresentada na Conferência de População, para o ano seguinte, 1984, no México. 1

Nesse processo, prevaleceu o discurso consensual baseado nos princípios do direito à saúde e na autonomia das mulheres e dos casais sobre a definição do tamanho da prole. Esse consenso construído entre governo e setores da sociedade civil teve como respaldo, no setor da saúde, o processo avançado da reforma sanitária em curso no país. Anos mais tarde, o Movimento da Reforma Sanitária contabilizaria sua grande conquista ao inscrever na Constituição Federal a saúde como direito do cidadão e o dever do Estado. O planejamento familiar, por sua vez, foi definido na Carta Magna, como de livre arbítrio das pessoas envolvidas.

Com o propósito de garantir autonomia na escolha dos métodos e do controle da fertilidade, são valorizadas as práticas de educação em saúde e sexualidade, entendidas como instrumentos disseminadores de informações para fortalecimento da autonomia. O Programa recomenda ainda que se ofereça o conjunto de tecnologias disponíveis para a anticoncepção e que a atenção ao planejamento familiar seja realizada no contexto da atenção à saúde, portanto sob as diretrizes do princípio da integralidade da saúde.7,8 O PAISM estabelece ainda que o planejamento familiar deva incluir ações para a anticoncepção e também atenção aos casos de infertilidade.

A consagração do direito ao planejamento familiar está explicitada no $\$ 7$ do Art. 226 da Constituição Federal, ${ }^{9}$ de 1988. No documento, estão estabelecidas as diretrizes a serem obedecidas pelo legislador ordinário, que não deve vincular direito e acesso aos serviços de planejamento familiar às políticas de controle demográfico. Entre essas diretrizes figuram claramente, a liberdade de decisão do casal e a responsabilidade do Estado em 
prover recursos educacionais e científicos para o exercício desse direito. A regulamentação do direito constitucional se deu pela Lei n 9263,10 de 12 de janeiro de 1996 representando um avanço na implementação do PAISM pelo Sistema Único de Saúde (SUS).

Ao recomendar o conjunto dos métodos anticoncepcionais disponíveis - naturais ou comportamentais; de barreira; hormonais; DIUs e laqueadura, o Ministério da Saúde reafirma a autonomia e a liberdade da escolha para as usuárias do planejamento familiar do SUS:

"Planejamento familiar é o direito que toda pessoa tem à informação, à assistência especializada e ao acesso aos recursos que permitam optar livre e conscientemente por ter ou não ter filhos. O número, o espaçamento entre eles e a escolha do método anticoncepcional mais adequado são opções que toda mulher deve ter o direito de escolher de forma livre e por meio da informação, sem discriminação, coerção ou violência. (Ministério da Saúde; 1999). 11

Pode se afirmar que a autonomia tem diversos significados, relacionados à autodeterminação, direito à liberdade, privacidade, escolha individual, livre vontade. 12 Essencialmente, autonomia é a capacidade de pensar, decidir e agir, com base no livre pensamento e decisão independente. 13 No entanto, a vontade e a capacidade não são suficientes para o pleno exercício da autonomia. A informação é o pressuposto inarredável para a escolha que o indivíduo realiza, no contexto de uma sociedade equilibrada. ${ }^{14}$ No caso do planejamento familiar, o exercício da autonomia depende também da oferta de alternativas contraceptivas, traduzidos na existência e disponibilidade dos métodos contraceptivos nos serviços de saúde.

Esse estudo tem o objetivo de verificar algumas condições relacionadas à possibilidade de autonomia das mulheres, analisando a situação da oferta de atenção ao planejamento familiar nos municípios que operam a rede de atenção do SUS, incluindo a oferta do conjunto dos métodos contraceptivos.

\section{Métodos}

Trata-se de um estudo ecológico, transversal de cunho exploratório. O inquérito foi realizado por meio de um questionário auto-aplicado aos secretários municipais de saúde, no período entre novembro de 2003 e março de 2004.

A amostra de municípios foi probabilística do tipo aleatória estratificada, sorteada, e tomou por base o cadastro municipal do Censo Demográfico de 2000 do Instituto Brasileiro de Geografia e Estatística (IBGE). 15 Os estratos foram definidos como a combinação entre a região geográfica e classes de tamanho do município (até 5000 habitantes; de 5001 a 10.000 ; de 10.001 a 20.000 ; de 20.001 a 50.000 e acima de 50.000). Essa técnica garante a todos os municípios brasileiros a mesma oportunidade de compor cada estrato da amostra. Para obter estimativas sobre o conjunto dos municípios, foi utilizada a técnica de expansão da amostra dos 627 respondentes por meio de fatores aplicáveis a cada estrato por região geográfica e por tamanho da população. Dessa forma, os resultados da população em estudo permitem inferências sobre 5507 municípios brasileiros. Para fins de cálculo de amostra, foram adotados o nível de confiança de $95 \%$ e a chance de erro de $5 \%$. O questionário foi enviado aos gestores por correio, fax ou meio eletrônico. Para atingir o conjunto de municípios previsto na amostra, na fase final da coleta de dados, foram realizadas ligações telefônicas aos gestores municipais, a fim de estimulá-los a responderem ao questionário.

Para responder ao questionário, o gestor, ou o profissional da Secretaria de Saúde por ele indicado, concordou com os termos da carta que lhe foi dirigida, que garantiam o sigilo dos dados municipais e informavam que tais dados receberiam tratamento estatístico de forma agrupada, de acordo com as variáveis independentes que orientaram a amostra. O projeto de pesquisa foi apreciado e aprovado pelo Comitê de Ética em Pesquisa da Secretaria de Saúde do Distrito Federal.

A oferta de serviços e de métodos contraceptivos, entendida como condição indispensável para o exercício da autonomia das mulheres em relação ao planejamento familiar, foi verificada por meio de perguntas aos gestores municipais, que informam sobre aspectos da qualidade da atenção e a oferta de ações e atividades, além de levantar a disponibilidade de insumos para a atenção ao planejamento familiar. Para conhecer os aspectos da qualidade da atenção em planejamento familiar relacionadas à prática da autonomia das mulheres, foram usadas duas variáveis: oferta de informação e educação em saúde para conhecimento do corpo, da sexualidade e dos métodos contraceptivos; e vínculo entre a seleção do método contraceptivo e a oportunidade das mulheres de obter informações para orientar sua escolha.

A oferta de atividades da atenção - consultas de planejamento familiar, práticas educativas, atendi- 
mento à infertilidade e a disponibilidade dos métodos contraceptivos - foi verificada quanto à estimativa declarada de cobertura de demanda populacional que a rede de serviços da secretaria atende.

Foi realizado um processo permanente de crítica aos questionários respondidos, visando à consistência das respostas. Na etapa subseqüente, após digitação dos dados, foi realizada ainda uma crítica da base de dados, visando à correção de digitação e à validação das informações coletadas.

Para o tratamento estatístico dos dados, foi usado o programa SPSS ${ }^{\circledR}$. A análise estatística dispôs resultados de frequiências simples e cruzadas, com dados absolutos e relativos. Foram utilizados os testes Qui-quadrado e "t" de Student, ou análise de variância, para verificação de independência entre variáveis qualitativas e a igualdade entre médias, respectivamente.

\section{Resultados e Discussão}

Os resultados foram processados e serão apresentados por meio de análise descritiva, freqüência e cruzamentos entre as variáveis dependentes e independentes (dimensão populacional do município e região geográfica).

Em virtude do caráter institucional deste estudo e pelo fato de ter sido realizado pelo Ministério da Saúde, há possibilidade de se apresentarem vieses nas respostas dos gestores municipais, tanto na declaração das prioridades quanto nas questões de análise dos serviços e da oferta, em decorrência das relações hierárquicas existentes nessas duas instâncias do SUS.
As atividades de planejamento familiar figuram no consenso das prioridades na atenção às mulheres: para $86,3 \%$ dos municípios brasileiros $(n=5156)$, é alta a prioridade à oferta de planejamento familiar. Apenas 3,1\% manifestam baixa prioridade a essa modalidade de atenção. A prioridade atribuída ao planejamento familiar está associada à região geográfica $\left(\chi^{2}=96,735 ; p<0,001 ; \mathrm{n}=5158\right)$ e também ao tamanho do município $\left(\chi^{2}=100,336 ; p<0,001\right.$; $\mathrm{n}=5,158)$.

A região Centro-Oeste apresenta proporcionalmente o maior percentual de municípios com baixa prioridade $(6,2 \%)$, contrastando com os $0,8 \%$ dos municípios da região Sul. Esses resultados configuram um quadro sugestivo de que o planejamento familiar está incluído nas prioridades do SUS. Esse quadro pode ser resultado das mudanças nas políticas de saúde da mulher, proporcionadas pelo PAISM e identificadas nos mecanismos adotados pelo Estado, como a Lei 9263 de 12 de janeiro de 1996 publicada no Diário Oficial da União em 15 de janeiro de 1996, que prescreve o planejamento familiar como direito de todo cidadão. Esses mecanismos desembocam em instrumentos normativos aplicáveis ao SUS, como no caso da Portaria do Ministério da Saúde SAS, de número 48, de 11 de fevereiro de 1999, para orientar sobre a organização do atendimento em planejamento familiar.

Por outro lado, a prioridade atribuída ao planejamento familiar não está associada à modalidade de gestão do SUS ( $\left.\chi^{2}=2,120 ; p=0,714 ; \mathrm{n}=4647\right)$, embora a cobertura atendida esteja claramente associada à modalidade de gestão $\left(\chi^{2}=134.718 ; p<0,001\right.$; $\mathrm{n}=4742$ ), na qual os 4074 municípios em Gestão Plena de Atenção Básica têm elevado percentual de

Tabela 1

Estimativa da cobertura atendida de consultas e orientações para o planejamento familiar por região geográfica. Brasil, 2004.

\begin{tabular}{|c|c|c|c|c|c|c|c|c|c|c|c|c|}
\hline \multirow[t]{3}{*}{ Região } & \multicolumn{12}{|c|}{ Estimativa da cobertura de consultas e orientações oferecidas para o planejamento familiar (\%) } \\
\hline & \multicolumn{2}{|c|}{$0 \mathrm{l}-\mathrm{-} 25 \%$} & \multicolumn{2}{|c|}{$251--50 \%$} & \multicolumn{2}{|c|}{$501--75 \%$} & \multicolumn{2}{|c|}{$75 \mathrm{l}--100 \%$} & \multicolumn{2}{|c|}{ Não atende } & \multicolumn{2}{|c|}{ Total } \\
\hline & $\mathrm{n}$ & $\%$ & $\mathrm{n}$ & $\%$ & $\mathrm{n}$ & $\%$ & $\mathrm{n}$ & $\%$ & $\mathrm{n}$ & $\%$ & $\mathrm{n}$ & $\%$ \\
\hline Centro-Oeste & 18 & 4,2 & 27 & 6,3 & 97 & 22,6 & 269 & 62,7 & 18 & 4,2 & 429 & 100,0 \\
\hline Nordeste & 62 & 3,8 & 193 & 11,9 & 378 & 23,4 & 913 & 56,5 & 70 & 4,3 & 1616 & 100,0 \\
\hline Norte & 52 & 10,3 & 62 & 12,2 & 165 & 32,5 & 185 & 36,5 & 43 & 8,5 & 507 & 100,0 \\
\hline Sudeste & 53 & 3,3 & 219 & 13,8 & 395 & 24,9 & 789 & 49,7 & 131 & 8,3 & 1587 & 100,0 \\
\hline Sul & 9 & 0,8 & 106 & 9,5 & 254 & 22,8 & 685 & 61,4 & 62 & 5,6 & 1116 & 100,0 \\
\hline Total & 194 & 3,7 & 607 & 11,6 & 1289 & 24,5 & 2841 & 4,1 & 324 & 6,2 & 5255 & 100,0 \\
\hline
\end{tabular}

$\left(\chi^{2}=204,342 ; p<0,001 ; n=5.255\right)$. 
atendimento da demanda, mesmo sem a autonomia de gestão. Essa situação pode ser decorrente das garantias oferecidas na entrega de métodos pelo Ministério da Saúde, que realiza compras centralizadas para esses municípios.

A oferta de planejamento familiar, medida pela informação fornecida pelos gestores sobre a estimativa de demanda atendida nos municípios, mostra que essa atividade é amplamente realizada em todo o país e está associada à região geográfica $\left(\chi^{2}=204,342 ; p<0,001 ; \mathrm{n}=5.255\right)$, como mostra a Tabela 1.

Nas regiões Norte e Sudeste, estão localizados os maiores percentuais de municípios que não atendem a essa modalidade, com percentuais de $8,5 \%$ e $8,3 \%$, respectivamente. No entanto, a cobertura acima de $75 \%$ de atendimento da demanda é declarada por $49,7 \%(n=789)$ dos municípios do Sudeste contra $36,5 \%(\mathrm{n}=185)$ do Norte. No total, $54,1 \%(n=2841)$ dos municípios brasileiros declaram atender a mais que $75 \%$ da demanda do planejamento familiar.

A rápida transformação do comportamento reprodutivo no Brasil, de alguma forma, é resultado da absorção pelas mulheres de valores difundidos sobre os benefícios de uma prole menor, valores esses coincidentes com os interesses do controle demográfico na redução da natalidade. A aspiração ao número menor de filhos, baseada nos benefícios relacionados ao acesso a bens de consumo e serviços assistenciais, submete a decisão individual a uma lógica de possibilidades: menos filhos, melhores cuidados. Essa condição para a decisão, transplantada do mundo da lógica racional, obscurece, de certa forma, os aspectos subjetivos na decisão das mulheres. 16

O movimento feminista, como importante ator no debate social sobre o planejamento familiar, reivindica direitos reprodutivos que assegurem às mulheres o direito de controlar os seus corpos, de optar por ter filhos ou não, e na quantidade desejada. Sendo assim, o movimento reivindica o acesso aos meios anticoncepcionais e, ao mesmo tempo, denuncia os abusos cometidos em nome da liberdade de contracepção: a falta de informação e de assistência médica, além do aumento abusivo das esterilizações cirúrgicas. 17

A posição oficial do Brasil na adoção da livre escolha dos casais pelo tamanho da prole reflete a influência do discurso que desvincula o controle da fertilidade do controle demográfico. Desta forma, estabeleceu-se para o país uma ética política para o planejamento familiar relacionada à autonomia. $\mathrm{O}$ princípio da autonomia ou da liberdade individual é
Tabela 2

Realização de práticas de educação em saúde no município, por região geográfica. Brasil, 2004.

\begin{tabular}{|c|c|c|c|c|c|c|}
\hline \multirow[t]{3}{*}{ Região } & \multicolumn{6}{|c|}{ Realização de práticas de educação em saúde } \\
\hline & \multicolumn{2}{|c|}{$\operatorname{sim}$} & \multicolumn{2}{|c|}{ Não } & \multicolumn{2}{|c|}{ Total } \\
\hline & $\mathrm{n}$ & $\%$ & $\mathrm{n}$ & $\%$ & $\mathrm{n}$ & $\%$ \\
\hline Centro-Oeste & 401 & 93,7 & 27 & 6,3 & 428 & 100,0 \\
\hline Nordeste & 1599 & 93,8 & 105 & 6,2 & 1704 & 100,0 \\
\hline Norte & 427 & 84,6 & 78 & 15,4 & 505 & 100,0 \\
\hline Sudeste & 1429 & 87,1 & 211 & 12,9 & 1640 & 100,0 \\
\hline Sul & 984 & 85,6 & 166 & 14,4 & 1150 & 100,0 \\
\hline Total & 4840 & 89,2 & 587 & 10,8 & 5427 & 100,0 \\
\hline
\end{tabular}

$\left(\chi^{2}=81,253 ; p<0,001 ; \mathrm{n}=5.427\right)$

Fonte: Costa AM. Atenção Integral à Saúde das Mulheres. Brasília (DF); 2004.23

Tabela 3

Realização de práticas de educação em saúde no município, por região geográfica. Brasil, 2004.

\begin{tabular}{|c|c|c|c|c|c|c|}
\hline \multirow[t]{3}{*}{ Região } & \multicolumn{6}{|c|}{ Realização de práticas de educação em saúde } \\
\hline & \multicolumn{2}{|c|}{ Sim } & \multicolumn{2}{|c|}{ Não } & \multicolumn{2}{|c|}{ Total } \\
\hline & $\mathrm{n}$ & $\%$ & $\mathrm{n}$ & $\%$ & $\mathrm{n}$ & $\%$ \\
\hline$<51$ & 1135 & 87,2 & 167 & 12,8 & 1302 & 100,0 \\
\hline $5 \vdash 101$ & 1141 & 87,2 & 158 & 12,2 & 1291 & 100,0 \\
\hline $10+20$ & 1207 & 87,8 & 166 & 12,0 & 1381 & 100,0 \\
\hline $20+50$ & 851 & 90,7 & 87 & 9,3 & 938 & 100,0 \\
\hline$>50$ & 506 & 98,3 & 9 & 1,7 & 515 & 100,0 \\
\hline Total & 4840 & 89,2 & 587 & 10,8 & 5427 & 100,0 \\
\hline
\end{tabular}

$\chi^{2}=56,453 ; p<0,001 ; n=5.427$

Fonte: Costa AM. Atenção Integral à Saúde das Mulheres. Brasília (DF); 2004.23

proposto por diversos autores como um dos princípios éticos para a regência da vida coletiva. A autonomia garante a todo indivíduo plena liberdade de decisão e de ação, desde que essa não interfira nos direitos de outras pessoas. ${ }^{18-20}$

Um dos requisitos para a autonomia na escolha do método contraceptivo é a informação adquirida nas práticas educativas. Portanto, a oferta dessas práticas pelos serviços é uma medida que informa sobre a autonomia das mulheres na seleção do método. A realização de práticas de educação e promoção da saúde para que as mulheres possam 
Estimativa de cobertura por tipo de método contraceptivo nos municípios do país. Brasil, 2004.

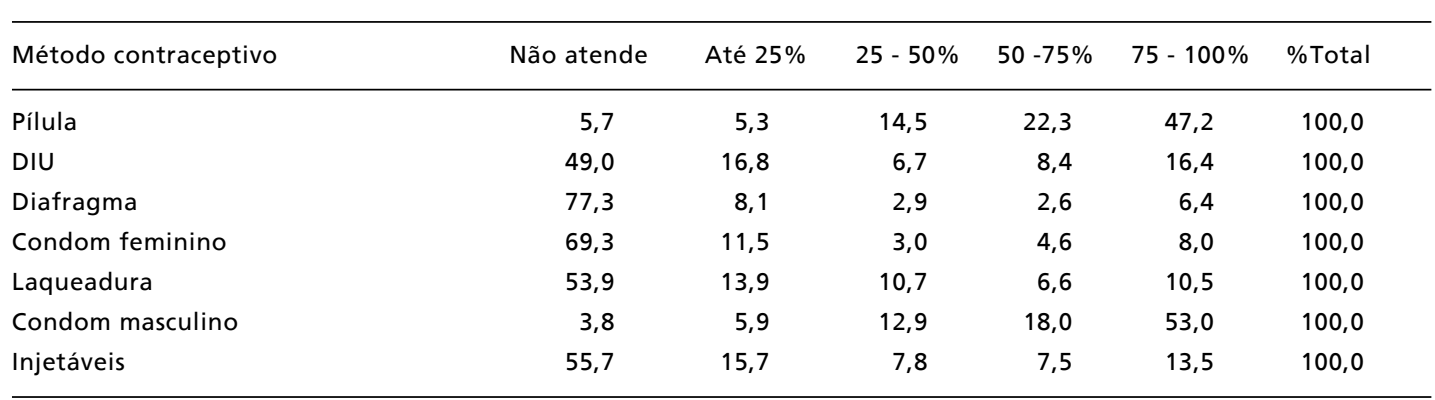

Fonte: Costa AM. Atenção Integral à Saúde das Mulheres. Brasília (DF); 2004.23

conhecer seu corpo e métodos contraceptivos está associada à região geográfica $\left(\chi^{2}=81,253 ; p<0,001\right.$; $\mathrm{n}=5427)$ e ao tamanho do município $\left(\chi^{2}=56,453\right.$; $p<0,001 ; \mathrm{n}=5427)$, conforme as Tabelas 2 e 3 .

Os resultados do estudo evidenciam que a oferta da informação relacionada ao conhecimento do corpo, à sexualidade e aos métodos contraceptivos não é realidade para os municípios menores: 98,3\% dos 515 municípios com mais de 50.000 habitantes realizam a educação em saúde com esse objetivo, enquanto 12,4\% 3974 dos municípios com até 20.000 habitantes não a realizam.

Assim, 89,2\% municípios afirmam oferecer práticas educativas $(\mathrm{n}=5427)$ visando ao conhecimento do corpo e dos métodos contraceptivos e, dos 587 municípios que não realizam essa prática, 83,6\% concentra-se nos de menor dimensão populacional, ou seja, até 20.000 habitantes. Esses dados sugerem que as práticas educativas são mais valorizadas e realizadas em municípios de maior dimensão populacional e em modalidade de Gestão Plena, pois, dos 4206 municípios beneficiados pela Gestão Plena de Atenção Básica, 10,4\% não oferecem práticas educativas, contra 3,8\% dos 685 municípios com Gestão Plena de Sistema o $\left(\chi^{2}=31,039 ; p<0,001 ; \mathrm{n}=4900\right)$.

A organização da atenção para o planejamento familiar deve garantir às mulheres informações claras e seguras que as orientem na escolha do método anticoncepcional. Isso significa que a oferta de práticas educativas deve ser valorizada na dinâmica do atendimento, a ponto de anteceder, preferencialmente, o momento da escolha. Na rotina dos serviços, esse ritual parece não existir para 39,6\% dos 2.160 municípios, que afirmam não conjugar as duas atividades. Entre esses, predominam os municípios pequenos: $43,7 \%$ dos municípios com menos de 20.000 habitantes. Essa situação coloca sob risco a prática da autonomia das mulheres no momento da escolha e seleção do método contraceptivo mais adequado, com informação e consciência.

$\mathrm{O}$ estabelecimento da dinâmica de atendimento de planejamento familiar que conjugue educação em saúde e consultas clínicas pode ter resultados importantes na adesão e na própria eficácia do método escolhido. Essa situação está associada à região geográfica $\left(\chi^{2}=264,776 ; p<0,001 ; \mathrm{n}=5455\right)$. Na região Nordeste, $74,1 \%$ dos 1691 municípios adotam essa rotina. Também está associada à modalidade de gestão $\left(\chi^{2}=23,521 ; p<0,001 ; \mathrm{n}=4919\right)$, uma vez que $66,8 \%$ dos 686 municípios em Gestão Plena de Sistema estabelecem a participação em reuniões educativas como pré-condição para que as usuárias do SUS selecionem o método anticoncepcional.

Outro aspecto importante na garantia da autonomia na escolha dos métodos contraceptivos é a sua disponibilidade no momento e lugar adequados. Preconiza-se que esteja disponível todo o conjunto de alternativas tecnológicas, cientificamente seguras, para que as mulheres não sejam conduzidas a um método pela ausência de oportunidade de escolher outro.

No Brasil, há uma polarização do uso de contraceptivos, na qual a pílula e a esterilização cirúrgica imperam. ${ }^{21}$ É desejável a reversão dessa situação, resultante, dentre outros aspectos, da indisponibilidade de tecnologias alternativas. Se essa reversão mudasse o quadro da distribuição do uso entre os distintos métodos contraceptivos, a autonomia das mulheres estaria mais próxima de ser atingida.

Os resultados deste estudo não são alentadores em relação à disponibilidade de outros métodos além da pílula, nos municípios brasileiros: a alta concentração da oferta de pílulas em detrimento da oferta de outras tecnologias alternativas é evidente conforme a Tabela 4.

O DIU e o diafragma estão indisponíveis na 
Estimativa de cobertura de atenção à infertilidade, por região geográfica. Brasil, 2004.

\begin{tabular}{|c|c|c|c|c|c|c|c|c|c|c|c|c|}
\hline \multirow[t]{3}{*}{ Região } & \multicolumn{12}{|c|}{ Estimativa da demanda que o município consegue atender: Atendimento à infertilidade (\%) } \\
\hline & \multicolumn{2}{|c|}{$0 \mathrm{l}--25 \%$} & \multicolumn{2}{|c|}{25 I- $50 \%$} & \multicolumn{2}{|c|}{50 I- $75 \%$} & \multicolumn{2}{|c|}{$75 \mid--100 \%$} & \multicolumn{2}{|c|}{ Não atende } & \multicolumn{2}{|c|}{ Total } \\
\hline & $\mathrm{n}$ & $\%$ & $\mathrm{n}$ & $\%$ & $\mathrm{n}$ & $\%$ & $\mathrm{n}$ & $\%$ & $\mathrm{n}$ & $\%$ & $\mathrm{n}$ & $\%$ \\
\hline Centro-Oeste & 53 & 11,9 & 54 & 12,1 & 18 & 4,0 & 27 & 6,1 & 294 & 65,9 & 446 & 100,0 \\
\hline Nordeste & 158 & 9,7 & 62 & 3,8 & 61 & 3,7 & 79 & 4,8 & 1274 & 78,0 & 1634 & 100,0 \\
\hline Norte & 43 & 8,5 & 9 & 1,8 & 9 & 1,8 & 18 & 3,6 & 427 & 84,4 & 506 & 100,0 \\
\hline Sudeste & 219 & 13,4 & 131 & 8,0 & 61 & 3,7 & 105 & 6,4 & 1114 & 68,3 & 1630 & 100,0 \\
\hline Sul & 149 & 13,2 & 44 & 3,9 & 61 & 5,4 & 87 & 7,7 & 791 & 69,9 & 1132 & 100,0 \\
\hline Total & 622 & 11,6 & 300 & 5,6 & 210 & 3,9 & 316 & 5,9 & 3900 & 72,9 & 5348 & 100,0 \\
\hline
\end{tabular}

$\left(\chi^{2}=147,204 ; p<0,001 ; \mathrm{n}=5.348\right)$

Fonte: Costa AM. Atenção Integral à Saúde das Mulheres. Brasília (DF); 2004.23

maior parte dos municípios e até mesmo a laqueadura tubária não é oferecida em 56,4\% (n=5261) deles, sugerindo que sua expressiva prática no país pode estar sendo realizada mediante o pagamento do procedimento. Por sua vez, é expressivo o volume de oferta do preservativo masculino, evidenciado pelos 56,6\% dos municípios que declaram atender acima de $75 \%$ da demanda, de um total de 5157. Esse dado requer estudos mais apurados, mas, provavelmente, esse resultado pode ter relação com o programa de AIDS, que investiu na disseminação do uso e na distribuição de camisinha masculina.

Embora os contraceptivos hormonais injetáveis tenham sido introduzidos no mercado brasileiro na década de 1990, são mais ofertados pelos municípios que os métodos de barreira do tipo diafragma ou camisinha feminina. $\mathrm{O}$ diafragma não é oferecido por $77,3 \%$ dos municípios $(n=5358)$ e apenas $6,4 \%$ desses atendem acima de $75 \%$ da demanda das mulheres por diafragma; já no caso da oferta dos injetáveis, 13,5 \% dos municípios $(n=5314)$ conseguem atender a mais que $75 \%$ da demanda. Tais dados fornecem indícios de que os gestores do SUS têm privilegiado a oferta, de forma diferenciada, dessas tecnologias contraceptivas hormonais.

O incentivo ao uso do diafragma no Brasil foi muito praticado pelo movimento feminista nos anos oitenta, sob os argumentos da inocuidade, do baixo custo e da promoção da autonomia da mulher. A classe médica, no entanto, nunca se aliou a essa intenção das mulheres feministas. $\mathrm{O}$ vigor da introdução dos injetáveis nos municípios sugere um reforço de tendência do uso de contraceptivos hormonais, da mesma forma como já vem ocorrendo com a pílula. Atualmente, envolvido com outras prioridades de sua luta, o movimento feminista já não reivindica e nem defende, como outrora, a oferta de métodos mais inócuos. Com isso, reduzem-se as chances de disseminar o diafragma como tecnologia contraceptiva de larga utilização no país.

A iniqüidade na oferta dos métodos contraceptivos evidencia-se na análise da distribuição por região e dimensão da população dos municípios. O diafragma não é oferecido em 92,9\% dos municípios da região Norte $(n=507)$ assim como o DIU, ausente em 76,3\% dos municípios ( $\mathrm{n}=524)$. Mais da metade dos 3959 municípios com até 20.000 habitantes não oferece o DIU. No caso do diafragma, mais de $80 \%$ dos 4867 municípios com população menor que 50.000 habitantes não oferecem esse método. Mais da metade $(54,9 \%)$, dos 490 municípios com população superior a 50.000 habitantes, também não oferecem o diafragma.

Mesmo no caso da pílula, que junto com a laqueadura lideram o ranking dos métodos mais usados no país, há uma importante discrepância de oferta entre a região Norte e as demais regiões. Dentre os 1115 municípios do Sul, $67,0 \%$ atendem a mais de $75 \%$ da demanda da pílula e apenas 33,4\% dos 497 municípios do Norte atendem a esse mesmo nível de demanda. Também é no Norte que $12,5 \%$ dos municípios não oferecem essa tecnologia, contra 3,9 $\%$ do Sul. Neste caso, observa-se também que nos municípios com população inferior a 20.000 habitantes a oferta de pílulas é menor.

A atenção aos casos de infertilidade, que deveria integrar o conjunto de atividades de planejamento familiar, não é realizada em 72,9\% $(n=5348)$ dos 
Estimativa de cobertura na atenção à Infertilidade por dimensão populacional dos municípios. Brasil, 2004.

População Estimativa da demanda que o município consegue atender: Atendimento à infertilidade (em milhares)

\begin{tabular}{|c|c|c|c|c|c|c|c|c|c|c|c|c|}
\hline & \multicolumn{2}{|c|}{0 a $25 \%$} & 25 & a $50 \%$ & \multicolumn{2}{|c|}{50 a $75 \%$} & 75 & a $100 \%$ & \multicolumn{2}{|c|}{ Não atende } & \multicolumn{2}{|c|}{ Total } \\
\hline & $n$ & $\%$ & $n$ & $\%$ & $n$ & $\%$ & $n$ & $\%$ & $n$ & $\%$ & $n$ & $\%$ \\
\hline$<51$ & 79 & 6,1 & 44 & 3,4 & 53 & 4,1 & 61 & 4,7 & 1056 & 81,7 & 1293 & 100,0 \\
\hline $5+101$ & 158 & 12,3 & 45 & 3,5 & 44 & 3,4 & 79 & 6,2 & 957 & 74,6 & 1283 & 100,0 \\
\hline $10+20$ & 184 & 13,6 & 70 & 5,2 & 61 & 4,5 & 61 & 4,5 & 979 & 72,3 & 1355 & 100,0 \\
\hline 20ㅏㅏ 50 & 141 & 15,3 & 70 & 7,6 & 35 & 3,8 & 61 & 6,6 & 613 & 66,6 & 920 & 100,0 \\
\hline$>50$ & 62 & 12,4 & 71 & 14,2 & 18 & 3,6 & 53 & 10,6 & 295 & 59,1 & 499 & 100,0 \\
\hline Total & 624 & 11,7 & 300 & 5,6 & 211 & 3,9 & 315 & 5,9 & 3900 & 72,9 & 5350 & 100,0 \\
\hline
\end{tabular}

$\left(\chi^{2}=206,051 ; p<0,001 ; n=5.350\right)$

Fonte: Costa AM. Atenção Integral à Saúde das Mulheres. Brasília (DF); 2004.23

municípios brasileiros e, em apenas 5,9\% deles, são atendidas demandas superiores a $75 \%$ (Tabela 5).

$\mathrm{Na}$ distribuição regional, são os seguintes percentuais de municípios que não dispõem dessa modalidade de atendimento: $84,4 \%$ dos municípios do Norte $(\mathrm{n}=506) ; 78,0 \%$ dos municípios do Nordeste $(\mathrm{n}=1634) ; 69,9 \%$ dos municípios do Sul $(\mathrm{n}=1132) ; 68,3 \%$ dos municípios do Sudeste ( $n=1630)$ e $65,9 \%$ dos municípios do Centro-Oeste $(n=446)$. A estimativa de atendimento à demanda nos casos de infertilidade está associada à região geográfica $\left(\chi^{2}=147,204 ; p<0,001 ; \mathrm{n}=5348\right)$ e ao tamanho do município $\left(\chi^{2}=206,051 ; p<0,001 ; \mathrm{n}=5350\right)$. Como verificado nas situações anteriores, os municípios com menor número de habitantes são os que deixam de oferecer atenção aos casos de infertilidade (Tabela 6).

Os resultados deste estudo, além de desvendarem situações que merecem futuros desdobramentos de investigação, revelam os desafios impostos ao SUS no atendimento às necessidades das mulheres em relação ao planejamento familiar e, especialmente, no cumprimento do princípio da autonomia como regente da política de integralidade na atenção e no cuidado a saúde das mulheres.

O sucesso das iniciativas favoráveis ao controle demográfico no Brasil nos últimos 30 anos pode ser celebrado pela notável redução da taxa de fecundidade: de 6,3 filhos por mulher, em 1960, para 2,3 filhos em 2000.15 Apesar do amplo debate, as mudanças que ocorreram não mobilizaram resistências significativas de nenhum segmento do Estado, incluindo a sociedade organizada e o movimento feminista.
A justificativa teórica da redução da pobreza, em defesa da diminuição do número de nascimentos, não se concretizou. Esse equívoco fortalece o argumento de que o desenvolvimento não pode ser alcançado pela exclusão da população pobre e de que o Estado deve oferecer alternativas contraceptivas articuladas em ações de saúde, dissociadas dos argumentos malthusianos.22 A subordinação da política externa brasileira aos interesses dos países que detêm a hegemonia no debate do controle da natalidade é histórica e revela-se por meio da queda de natalidade ocorrida nas três últimas décadas. $1,2,5-7$

\section{Conclusões}

Os resultados deste estudo conformam evidências de que tema do planejamento familiar no Brasil ainda não está superado como objeto de políticas públicas. $\mathrm{O}$ fato de os interesses pelo controle populacional constituírem prioridade para o Estado Neoliberal renova o debate político, tal como o foi nas décadas passadas, quando o enfrentamento desse tema manifestou-se no debate social. Mesmo quando os governantes reafirmavam, no discurso, a soberania do país, nunca foram operadas nas práticas dos serviços mudanças que pudessem garanti-la. O expediente do Estado em formular uma política de Planejamento Familiar integrada às políticas de saúde, por meio do PAISM, teve o mérito de dissociar sua prática do controle demográfico, saneando e neutralizando as resistências polarizadas. 
As restrições do acesso e da oferta aos serviços de planejamento familiar apresentadas no presente estudo mostram ainda carência de garantias elementares na sua prática. O processo de implantação do planejamento familiar no SUS requer a superação de problemas relativos à oferta de métodos contraceptivos, assim como o aperfeiçoamento das atividades e da organização dos serviços no que diz respeito às práticas educativas; às consultas clínicas e, especialmente, à oferta de atenção à infertilidade.

Quanto à organização da atenção prestada às mulheres, ainda há um longo caminho a percorrer para que seja possível garantir-lhes informações e tecnologias para o exercício de suas escolhas reprodutivas autônomas. Por enquanto, a autonomia decisória das mulheres é refém da oferta de serviços de atenção e de métodos. Frente a tais resultados, o questionamento fundamental que se impõe para os formuladores de políticas de saúde, gestores, movi-

\section{Referências}

1. Fonseca Sobrinho D. Estado e população: uma história do planejamento familiar no Brasil. Rio de Janeiro: CEDEPLAR; 1993.

2. Pêgo RA, Richa AC. Estado e instituições de planejamento familiar. In: Controle da natalidade x planejamento familiar no Brasil. Rio de Janeiro: Achiamé; 1987. p. 31-41.

3. Rocha MIB. Política demográfica e parlamento. Debates e decisões sobre o controle da natalidade [tese doutorado]. Campinas: Núcleo de Estudos da População da Universidade Estadual de Campinas; 1993.

4. Brasil. Congresso. Relatório final da Comissão Parlamentar Mista de Inquérito destinada a examinar a incidência de esterilização em massa de mulheres no Brasil. Brasília (DF); 1993

5. Costa AM. Planejamento familiar no Brasil. 1995. Disponível em URL: http://www.cfm.org.br/revista/bio2v4/ planeja.html [2004 jun 04].

6. Pêgo RA. A luta das mulheres pela livre concepção. In: Controle da natalidade $x$ planejamento familiar no Brasil. Rio de Janeiro: Achiamé; 1987. p. 61-9

7. Costa AM. PAISM: uma política de assistência integral à saúde da mulher a ser resgatada. São Paulo: Comissão de Cidadania e Reprodução; 1992.

8. Ministério da Saúde. Assistência integral à saúde da mulher: bases para uma ação programática. Brasília (DF): 1984. (Textos Básicos de Saúde, Série B, v. 6).

9. Brasil. Constituição (1988). Constituição Federal. Brasília (DF): Câmara dos Deputados, Coordenação de Publicações; 2002 . mentos sociais, incluindo o movimento feminista, e pesquisadores é se o Brasil está conseguindo cumprir a agenda definida em sua política demográfica nãocontrolista, que deveria promover a livre escolha em relação à reprodução, ou apenas está implementando, de forma disciplinada e dissimulada, a política e os interesses internacionais de redução da população brasileira.

\section{Agradecimentos}

Ao apoio oferecido pela CAPES do Ministério da Educação e Cultura por ter propiciado Bolsa Parcial de Doutorado à primeira autora assim como à Ford Foundation, pelo apoio financeiro ao desenvolvimento da linha de pesquisa de Avaliação da Atenção Integral à Saúde da Mulher, permitindo a definição da metodologia usada neste estudo.

10. Brasil. Lei $n^{\circ} 9.263$, de 12 de janeiro de 1996. Regula o $\$ 7^{\circ}$ do artigo 226 da Constituição Federal, que trata do planejamento familiar, estabelece penalidades e dá outras providências. Disponível em URL: http://www.pge.sp.gov. br/centrodeestudos/tecavirtual/dh/ volume\%20i/plajlei9263.htm [2004 jun 4].

11. Ministério da Saúde. Portaria $n^{\circ} 48$, de 11 de fevereiro de 1999. Ano Internacional da Mulher. Brasília (DF); 2004.

12. Beauchamp TL, Childress JF. Principles of biomedical ethics. 4. ed. New York: Oxford; 1994.

13. Almeida M, Muñoz DR. A responsabilidade médica: uma visão bioética. Disponível em URL http://www.unifesp.br/ dpato/medlegal/respmed.htm. [2005 jun. 4]

14. Marchi MM. Autonomia e heteronomia na relação entre profissional de saúde e usuário dos Serviços de Saúde. Rev Bioética 1; v. 6 [on line]. Disponível em URL http:// www.portalmedico.org.br/revista/bio1v6/autoheter.htm (2005 jun 23)

15. IBGE (Instituto Brasileiro de Geografia e Estatística). Cadastro de municípios. Censo Demográfico: 2000. Disponível em URL: http://www.ibge.gov.br/home/estatistica/populacao /censo2000/default.shtm. [2004 jul 05].

16. Costa AM. Metáforas do desejo: a esterilização como processo de defesa. Rev Ciências Med 2003; 12: 3-13.

17. SOS Corpo. Grupo de Saúde da Mulher. Viagem ao mundo da contracepção: um guia sobre os métodos contracepcionais. Rio de Janeiro: Rosa dos Tempos; 1991.

18. Cohen C, Segre M. Breve discurso sobre valores, moral, eticidade e ética. Rev Bioética 1994; 2: 19-24. 
19. Isaacs SL. Incentives population policy and reproductive rights: ethical issues. Stud Fam Plann 1995; 26: 363-7.

20. Macklin R. Liberty, utility and justice: an ethical approach to unwanted pregnancy. Int J Gyncol Obstet 1989; 3: 37-49.

21. Berquó E. Brasil, um caso exemplar: anticoncepção e partos cirúrgicos - à espera de uma ação exemplar. Rev Est Feministas 1993; 1: 366-81.

22. Stephan-Souza AI. Relendo a política de contracepção: o olhar de um profissional sobre o cotidiano das Unidades Públicas de Saúde. Cad Saúde Pública 1995; 11: 408-24.
23. Costa AM. Atenção Integral à Saúde das Mulheres: quo vadis? Uma avaliação da integralidade na Atenção à Saúde das Mulheres no Brasil [tese doutorado]. Brasília (DF): Faculdade de Ciências da Saúde da Universidade de Brasília; 2004.

Recebido em 6 de abril de 2004

Versão final apresentada em 1 de fevereiro de 2006

Aprovado em 9 de fevereiro de 2006 